\title{
ESTUDO DOS FATORES DE RISCO PARA O DESENVOLVIMENTO DE DOENÇAS CRÔNICAS NÃO TRANSMISSÍVEIS ENTRE FUNCIONÁRIOS
}

\author{
Carlos A. Zamai \\ Alan A. Rodrigues \\ Antonia D. P. Bankoff \\ Marcelo Filocomo \\ Luiz Ernani S. Braga \\ José Antonio S. Barbosa
}

\section{Resumo}

O objetivo deste estudo foi avaliar o estado nutricional e fatores de risco para doenças crônicas não transmissíveis em 250 funcionários. Foram estudadas as variáveis: estado nutricional, circunferência da cintura, pressão arterial, glicemia, idade e nível de escolaridade. Verificou-se sobrepeso e obesidade em $45 \%$ e $10 \%$ da amostra, respectivamente, com associação significativa entre o estado nutricional e grupo sexual, mas não entre estado nutricional e nível de escolaridade, ajustados por idade. Verificaram-se valores de circunferência da cintura elevados em 33,0\% dos funcionários, com diferença significativa entre grupos sexuais. Observou-se que a pressão arterial sistólica (PAS) e pressão arterial diastólica (PAD) estavam elevadas em $18 \%$ e $11 \%$ dos funcionários, respectivamente, com associação significativa entre sexos após ajuste por idade. Diagnosticou-se diabetes mellitus tipo II em $2,5 \%$ da amostra e glicemia alterada em $4 \%$. Após ajuste por idade, as únicas variáveis associadas à circunferência da cintura foram a PAS e a PAD. Estes resultados permitiram que atividades preventivas e educacionais fossem desenvolvidas e implantadas pela empresa, de modo a melhorar a qualidade de vida e a produtividade dos funcionários.

\section{Palavras-Chaves}

Fatores de risco; Doença crônica; Funcionários.

\section{STUDY OF RISK FACTORS FOR THE DEVELOPMENT OF CHRONIC DISEASES NOT TRANSFEREIBLE AMONG EMPLOYEES OFFICIALS}

Carlos A. Zamai

Alan A. Rodrigues

Antonia D. P. Bankoff

Marcelo Filocomo

Luiz Ernani S. Braga

José Antonio S. Barbosa

\begin{abstract}
The objective of this study was to evaluate the nutritional status and risk factors for chronic no transfereible diseases in 250 factory employees. The following variables were studied: nutritional status, waist circumference, blood pressure, blood glucose levels, age and education level. Overweight and obesity were observed in $45 \%$ and $10 \%$ of the sample, respectively, with a significant association between nutritional status and sexual group, but not between nutritional status and education level,
\end{abstract}


adjusted for age. The proportion of high waist circumference was 33\%, with a significant difference between sexual groups. It was observed that the systolic blood pressure (SBP) and diastolic blood pressure (DBP) they were elevated in the employees' $18 \%$ and $11, \%$, respectively, with significant association among female and male after age adjustment. It was diagnosed mellitus diabetes type II in $2,5 \%$ of the sample and altered glicemia in $4 \%$. After age adjustment, the only associated variables in the waist circumference were the SBP and DBP. These results allowed preventive and education activities to be developed and implanted by the company, in way to improve the life quality and the employees productivity.

\section{Key- Works}

Risk factors; Chronics diseases; Officials. 


\section{INTRODUÇÃO}

Os fatores de risco e as doenças crônicas não transmissíveis (DCNT), representam atualmente um importante problema de saúde pública no Brasil e no mundo, um quadro sanitário em que se combinam as doenças ligadas à pobreza típicas dos países em desenvolvimento e as doenças não transmissíveis dos países mais afluentes, refletindo as contradições do nosso processo de desenvolvimento. (ZAMAI et al., 2004, 2005).

A obesidade, por exemplo, representa o problema nutricional de maior ascensão entre a população observado nos últimos anos, sendo considerada uma epidemia mundial, presente tanto em países desenvolvidos como naqueles em desenvolvimento. (MONTEIRO et al. 1995; BLUMENKRANTZ, 2006).

Salientam Francischi et al. (2000) e Lerario (2002) que as tendências de transição nutricional decorrente da urbanização e industrialização ocorridas neste século direcionam para uma dieta mais ocidentalizada, com especial destaque para o aumento da densidade energética, maior consumo de carnes, leite e derivados ricos em gorduras, e redução do consumo de frutas, cereais, verduras e legumes, a qual, aliada à diminuição progressiva da atividade física, converge para o aumento no número de casos de obesidade em todo o mundo.

$\mathrm{Na}$ opinião de Duncan et al. (1993) as doenças crônicas não transmissíveis, como doenças cardiovasculares, câncer, diabetes mellitus e hipertensão arterial, compõem um grupo de entidades que se caracterizam por apresentar, de uma forma geral, longo período de latência, tempo de evolução prolongado, lesões irreversíveis e complicações que acarretam graus variáveis de incapacidade ou óbito, e vem ocupando um maior espaço no perfil de morbi-mortalidade de populações latino americanas. Em estudos recentes do Banco Mundial, as doenças crônicas não transmissíveis são responsáveis por uma taxa de 5 e 9 vezes maior de morte prematura do que as doenças transmissíveis e taxas 10 e 5 vezes maiores de incapacidade, em homens e mulheres, respectivamente. (ORGANIZAÇÃO, 2004).

A Sociedade Brasileira de Cardiologia (2005) e o Instituto Brasileiro de Geografia e Estatística (IBGE, 2006) apontam que $80 \%$ da população adulta é sedentária e que 52\% dos adultos brasileiros estão acima do peso, sendo $11 \%$ obesos, o que explica o aumento da morbidade e mortalidade, já que a obesidade é fator de risco para várias doenças crônicas não transmissíveis. As maiores proporções de excesso de peso 
e obesidade concentram-se na Região Sul do país, prevalecendo em $89,6 \%$ e $25,2 \%$ da população, respectivamente.

Zamai et al. (2007) salientam ainda que o sedentarismo não representa apenas um risco pessoal de enfermidades, mas tem um custo econômico alto para o indivíduo, para sua família e para os cofres públicos.

Em seu estudo Castanheira (2003) aponta que é a localização abdominal de gordura que se mostra mais associada a distúrbios metabólicos e riscos cardiovasculares como dislipidemias, hipertensão arterial e diabetes mellitus. Medidas regionais de obesidade, entre as quais a circunferência da cintura, são capazes de fornecer estimativas de gordura centralizada que, por sua vez, está relacionada à quantidade de tecido adiposo visceral (LENARIO et al., 2002). Assim, essas medidas vêm sendo largamente utilizadas em estudos de base populacional como indicadores da gordura abdominal, seja pela sua associação com a ocorrência de doenças cardiovasculares como, por exemplo, a hipertensão arterial, seja pela alta correlação que possuem com métodos laboratoriais de avaliação da composição corporal.

A obesidade, particularmente aquela localizada na região abdominal, pode elevar o risco da ocorrência de diabetes tipo II em dez vezes, fato este que tem aumentado de forma exponencial em vários países, inclusive no Brasil. (BLUMENKRANTZ, 2006; SARTORELLI, 2003).

De acordo com Brasil (2002), o diabetes mellitus responde por cerca de 25 mil óbitos anuais, sendo classificado como a sexta causa de morte no país. Segundo Jung (1997), em torno de 75\% dos pacientes diabéticos não dependentes de insulina estão acima do peso desejável, e para aumento de $10 \%$ no peso corporal, há aumento de 2mg/dL na glicemia em jejum. (BLUMENKRANTZ, 2006).

Ainda na opinião deste autor a hipertensão arterial, a qual está associada a fatores familiares, genéticos e ambientais e que acomete jovens adultos de 20 a 45 anos, prevalece seis vezes mais em obesos do que em não obesos. O aumento de $10 \%$ na gordura corporal reflete aumento significativo da pressão arterial. (FRANCISCHI et al., 2000, SOCIEDADE, 2004).

O diabetes mellitus e a hipertensão arterial associados aumentam consideravelmente o risco de doenças cardiovasculares, que representam a primeira causa de óbito no país e são responsáveis por elevadas taxas de internação hospitalar e incapacitação física. (BRASIL, 2002). 
A realização desta investigação tem como proposta avaliar o estado nutricional e identificar os principais fatores de risco para o desenvolvimento de doenças crônicas não transmissíveis em um grupo de colaboradores pertencentes a uma indústria de Campinas.

\section{OBJETIVO GERAL}

O objetivo deste estudo foi avaliar o estado nutricional e fatores de risco para doenças crônicas não transmissíveis em 250 funcionários.

\section{METODOLOGIA}

Para o desenvolvimento deste trabalho foram coletados peso, estatura e circunferência da cintura, onde também se anotou os dados referentes à idade e à escolaridade dos mesmos. Em cada um dos participantes, verificou-se a pressão arterial e foi determinada a glicemia capilar em jejum.

Após concordância os sujeitos foram pesados antes da refeição, descalços e com o mínimo de vestimentas, em balança antropométrica Filizola, com capacidade máxima de 150kg e subdividida em 100g. A estatura foi verificada com o auxílio de um estadiômetro tipo trena, marca Seca ${ }^{\circ}$, estando o sujeito em posição ereta, com os pés juntos, mãos ao lado do corpo e cabeça posicionada num ângulo de $90^{\circ}$. Para a avaliação do estado nutricional, utilizou-se a classificação do índice de massa corporal (IMC) com os pontos de cortes da Organização Mundial da Saúde (1997).

Os sujeitos foram então classificados como eutróficos, com sobrepeso e obesos. A medida da circunferência da cintura foi obtida de acordo com Cameron (1984), com o auxílio de uma fita métrica extensível, de material resistente, com precisão de $0,1 \mathrm{~cm}$ e $150 \mathrm{~cm}$, estando o indivíduo despido nesta região, em pé, com os pés juntos, os braços estendidos lateralmente e o abdômen relaxado. Para identificar excesso de massa de gordura na região do tronco utilizou-se o ponto de corte sugerido por Han et al. (1995).

A pressão arterial foi aferida através de um esfigmomanômetro (medidor de pressão arterial) da marca Tycos. Os sujeitos foram previamente orientados a não fumar, beber café ou se alimentar nos 60 minutos antes das aferições. Para evitar variações acentuadas nos valores obtidos, a pressão arterial foi aferida após 5 a 10 minutos de repouso e relaxamento na sala de avaliação física. Para a verificação, os sujeitos ficaram sentados, com o braço direito repousado sobre uma superfície firme à altura do coração, livre de 
roupas, com a palma da mão voltada para cima e o cotovelo ligeiramente fletido. Os critérios diagnósticos e de classificação adotados foram os recomendados pela Sociedade Brasileira de Hipertensão (2003).

As análises estatísticas foram realizadas com auxílio do programa Statistic 5,0. Para testar as associações entre variáveis de classificação, utilizou-se o qui-quadrado, e a razão de chances foi adotada no caso de associações entre duas variáveis binominais.

Após concordância os sujeitos da pesquisa assinaram o termo de consentimento livre e esclarecido, no qual constam todas as etapas do estudo. O presente estudo foi aprovado pela Comissão de Ética da Universidade, de acordo com a Resolução nº 196/96 do Conselho Nacional de Saúde.

\section{RESULTADOS}

A média de idade foi de $30,96 \pm 8,63$ anos, sendo $27,6 \%$ destes do sexo feminino e $72,4 \%$ do sexo masculino.

Em relação ao estado nutricional, 45\% da amostra apresentava sobrepeso (IMC ¡Ý $25 \mathrm{~kg} / \mathrm{m} 2$ ) e constatouse obesidade (IMC ¡Ý 30kg/m2) em 9,6\%. Quando divididos em grupos etários, verificou-se, após análise por razão de chances, que os indivíduos entre 21 e 39 anos tinham 2,34 vezes mais chances de apresentarem sobrepeso ou obesidade quando comparados aos menores de 20 anos (IC: 1,79-3,07). Os indivíduos acima de 40 anos, por sua vez, apresentaram 5,49 vezes mais chances de não estarem eutróficos em relação aos menores de 20 anos (IC: 3,21-9,40).

Os percentuais de sobrepeso e obesidade segundo sexo foram, respectivamente, 49,3\%, 40,4\% e $10,3 \%$ para o sexo masculino e $69,7 \%, 22,5 \%$ e $7,8 \%$ para o feminino, com associação significativa entre os $\operatorname{sexos}(\div 2=43,37 ; \mathrm{p}=0,000)$.

Não se observou associação entre a escolaridade e o estado nutricional dos indivíduos $(\div 2=1,08 ; \mathrm{p}=$ 0,77; IC: 0,96-1,19) após o ajuste por idade.

Os valores médios de circunferência da cintura foram de $81,37 \pm 9,49 \mathrm{~cm}$ e $89,01 \pm 9,88 \mathrm{~cm}$, para mulheres e homens, respectivamente. Encontrou-se circunferência da cintura elevada em 33\% da população, sendo as prevalências de risco para o desenvolvimento de doenças metabólicas associadas à obesidade de $49 \%$ entre as mulheres e de $26 \%$ entre homens, com diferença estatisticamente significativa $(\div 2=63,8 ; \mathrm{p}=$ $0,0000)$.

Conexões: revista da Faculdade de Educação Física da UNICAMP, Campinas, v. 6, n. 1, p. 14-30, jan/abr. 2008 
Em relação à pressão arterial, os valores médios encontrados para a pressão arterial sistólica (PAS) e a pressão arterial diastólica (PAD) foram 120,24 $\pm 12,18 \mathrm{mmHg}$ e 78,01 $\pm 10,56 \mathrm{mmHg}$, respectivamente. Constatou-se PAS elevada em $18 \%$ da amostra, enquanto a PAD apresentou-se elevada em $11 \%$ do grupo. A análise estatística por razão de chances, ajustada por idade, demonstrou que os homens da amostra estudada têm 3,14 vezes mais chance de apresentar PAS elevada e 2,27 vezes mais chance de apresentar PAD elevada em relação às mulheres.

Tabela 1- Associação entre o estado nutricional e as variáveis pressão arterial sistólica (PAS) e pressão arterial diastólica (PAD) elevadas e diabetes mellitus II/alteração na glicemia de jejum. (\%).

\begin{tabular}{|c|c|c|c|c|c|c|c|c|}
\hline \multirow[t]{2}{*}{ Variáveis } & \multicolumn{3}{|c|}{ Índice de Massa Corporal } & \multirow[t]{2}{*}{$\chi^{2}$} & \multirow[t]{2}{*}{$\mathbf{P}$} & \multirow{2}{*}{$\begin{array}{l}\text { OR }(\mathrm{IC})^{*} \\
\text { estróficos } \\
\text { sobrepeso }\end{array}$} & \multicolumn{2}{|r|}{ OR (IC)* } \\
\hline & Eutrófico & Sobrepeso & Obesidade & & & & $\mathbf{x}$ & $\begin{array}{l}\text { estróficos } \\
\text { obesidade }\end{array}$ \\
\hline \multicolumn{9}{|c|}{ PAS elevada } \\
\hline Sim & 10,3 & 25,1 & 40,0 & 48,36 & 0,000 & $2,19(1,75-2,72)$ & & $4,78(3,07-7,43)$ \\
\hline Não & 89,7 & 75,9 & 60,0 & & & & & \\
\hline \multicolumn{9}{|c|}{ PAD elevada } \\
\hline Sim & 5,1 & 15,8 & 27,5 & 38,39 & 0,000 & $2,32(1,77-3,03)$ & & $5,40(3,16-9,20)$ \\
\hline Não & 94,9 & 84,2 & 72,5 & & & & & \\
\hline \multicolumn{9}{|c|}{$\begin{array}{l}\text { Diabetes } \\
\text { Melitus } \\
\text { II/Alteração } \\
\text { na glicemia } \\
\text { de jejum }\end{array}$} \\
\hline Sim & 5,0 & 8,1 & 5,8 & 0,03 & 0,85 & $1,03(0,72-1,47)$ & & $1,06(0,52-1,17)$ \\
\hline Não & 95,0 & 91,9 & 94,2 & & & & & \\
\hline
\end{tabular}

Na Tabela 1 verifica-se a associação entre o estado nutricional e as variáveis PAS e PAD elevadas e diabetes mellitus II ou alteração na glicemia de jejum. Observou-se associação estatisticamente significativa entre o estado nutricional e as PAS e PAD elevadas $(\div 2=48,36$; e $p=0,000 ; \div 2=38,39$ e $p$ $=0,000$, respectivamente), com razões de prevalências similares (Tabela 1). Quando testada a associação entre o estado nutricional e o diabetes mellitus II ou alteração na glicemia de jejum, não se constatou diferença estatisticamente significativa $(\div 2=0,03 ; \mathrm{p}=0,85)$, mesmo com o ajuste por idade. As associações entre a classificação da circunferência da cintura e as prevalências de PAS e PAD elevadas, bem como de diabetes mellitus II ou alteração na glicemia de jejum, foram testadas levando-se em considerações possíveis efeitos da idade e do sexo (Tabela 2). 
Tabela 2- Associação entre a circunferência da cintura e as variáveis pressão arterial sistólica (PAS) e pressão arterial diastólica (PAD) elevadas e diabetes mellitus II/alteração na glicemia de jejum. (\%).

\begin{tabular}{|c|c|c|c|c|c|c|}
\hline \multirow[t]{3}{*}{ Variáveis } & \multicolumn{2}{|c|}{ Circunferência da cintura $(\mathrm{cm})$} & \multirow[t]{2}{*}{$\chi^{2 *}$} & \multirow[t]{2}{*}{$\mathbf{P}$} & \multirow[t]{2}{*}{$\mathbf{O R}$} & \multirow[t]{2}{*}{$\mathbf{O R}$} \\
\hline & $<$ & $\geq$ & & & & \\
\hline & $\begin{array}{l}80 \text { (mulheres)/ } \\
<94 \text { (homens) }\end{array}$ & 80(mulheres)/ & & & & \\
\hline \multicolumn{7}{|c|}{ PAS elevada } \\
\hline $\operatorname{Sim}$ & 6,9 & 19,5 & 40,71 & 0,0000 & 3,62 & $2,44-5,38$ \\
\hline Não & 93,1 & 80,5 & & & & \\
\hline \multicolumn{7}{|c|}{ PAD elevada } \\
\hline Sim & 12,8 & 21,8 & 46,77 & 0,0000 & 3,10 & $2,24-4,30$ \\
\hline Não & 87,2 & 71,2 & & & & \\
\hline \multicolumn{7}{|c|}{$\begin{array}{l}\text { Diabetes } \\
\text { Melitus } \\
\text { II/Alteração } \\
\text { na glicemia } \\
\text { de jejum }\end{array}$} \\
\hline $\operatorname{Sim}$ & 94,3 & 92,9 & 0,02 & 0,87 & 1,04 & $0,62-1,76$ \\
\hline Não & 5,7 & 7,1 & & & & \\
\hline
\end{tabular}

* Ajuste por idade e sexo.

Verifica-se associação significativa entre a classificação da circunferência da cintura e as prevalências de PAS elevada $(\div 2=46,77 ; \mathrm{p}=0,000)$ e de PAD elevada $(\div 2=40,71 ; \mathrm{p}=0,000)$, não sendo observada variação na razão de prevalências entre a PAS e a PAD.

Comparando-se essas razões com aquelas observadas para o estado nutricional, verifica-se uma tendência de as razões para a classificação da circunferência da cintura serem maiores do que para o estado nutricional. Não foi observada diferença estatisticamente significativa quando avaliada a associação entre o diabetes mellitus ou alteração na glicemia de jejum e a circunferência da cintura $(\div 2=0,02 ; p=0,87)$.

\section{DISCUSSÃO E CONCLUSÃO}

No Brasil em 2003, o excesso de peso já afetava 41,1\% dos homens e 40\% das mulheres, sendo a obesidade predominante em 8,9\% dos homens e 13,1\% das mulheres adultas do país. Assim, os obesos representavam $20 \%$ do total de homens e um terço das mulheres com excesso de peso. (INSTITUTO, 2006). 
Com base nos dados antropométricos obtidos neste estudo, pôde-se verificar alta prevalência de sobrepeso e obesidade, refletindo o quadro atual encontrado em pesquisas realizadas no Brasil. Em estudo realizado por Matos et al. (2004) avaliando a prevalência dos fatores de risco para doença cardiovascular em funcionários da Petrobrás, verificou-se prevalências de $42 \%$ de sobrepeso e de $17 \%$ de obesidade, ou seja, 59\% da amostra apresentavam excesso de peso. Souza et al. (2003), ao estudarem a obesidade e o risco cardiovascular de adultos, utilizando os critérios de diagnóstico da OMS, encontraram uma prevalência de obesidade de $17,8 \%$.

Em outros estudos realizados no Brasil com características semelhantes também encontraram prevalências elevadas de obesidade, como aqueles conduzidos por Gus et al. (2002), no Rio Grande do Sul, com 18,6\%, e por Cervato et al. (1997), em São Paulo, com 38\%. Em estudo realizado por Castanheira et al. (2003) com adultos no Sul do Brasil, verificou-se que mais da metade da população masculina estudada apresentou excesso de peso, sendo $16 \%$ obesos, resultados semelhantes aos obtidos no presente estudo, no qual 40,9\% dos homens apresentaram sobrepeso e, em 10,3\% deles, verificou-se obesidade.

Estudando a prevalência de obesidade em adultos e seus fatores de risco, Gigante et al. (1997) constataram que $21 \%$ da amostra apresentou obesidade, enquanto quase $40 \%$ apresentaram sobrepeso. A prevalência de obesidade foi significativamente mais elevada em mulheres que em homens (25\% e 15\%), em contraposição aos achados do presente estudo, em que o excesso de peso prevaleceu entre os homens, que apresentaram 2,76 vezes mais chances de apresentarem sobrepeso ou obesidade em comparação às mulheres.

De acordo com a Pesquisa de Orçamentos Familiares (POF 2002/2003), o excesso de peso tende a aumentar com a idade, de modo mais rápido para os homens e de modo mais lento, porém mais prolongado, para as mulheres 8. Este resultado também foi obtido no presente estudo, em que os indivíduos maiores de quarenta anos apresentaram 5,49 vezes mais chances de não estarem eutróficos em relação aos menores de 20 anos. Semelhantemente, Santana et al. (2001), em seu trabalho com 1.237 homens com idade de 14 a 76 anos, verificaram que 24,7\% da população estudada apresentou sobrepeso $(\mathrm{IMC} ; Y ́$ ' $25 \mathrm{~kg} / \mathrm{m} 2)$ e que essa prevalência aumentou com a idade, variando de 10,4\% no grupo de 14 a 25 anos para 26,4\% no grupo de 26 a 32 anos, e até 38\% acima de 32 anos de idade. Souza et al. (2003) 
observaram também em seu estudo que a prevalência de obesidade aumentou com a idade, sendo cerca de duas vezes mais elevada a partir dos trinta anos do que no grupo com idade entre 18 e 29 anos.

Estes autores ainda destacam em seu trabalho que a proporção de obesidade é cerca de quatro vezes mais elevada após os quarenta anos, fato que corrobora os resultados do presente estudo. Estes autores notaram, ainda, que as prevalências de obesidade são semelhantes nos dois sexos até os quarenta anos, mas, após esta idade, as mulheres passam a apresentar prevalência duas vezes mais elevada do que os homens.

Quanto à escolaridade, Wardle et al. (2002) investigaram os fatores sócio-econômicos preditivos de obesidade em mais de 15 mil homens e mulheres e observaram que o risco de se desenvolver obesidade é maior em indivíduos de menor escolaridade. Os autores verificaram que as pessoas que abandonaram os estudos mais jovens eram mais obesas que aquelas que continuaram estudando. Destaca-se ainda que Machado e Sichieri (2002), analisando a relação cintura-quadril e fatores de dieta em adultos, também observaram grande influência da baixa escolaridade sobre a distribuição de gordura abdominal, que pode ser indicativa do estado nutricional. Por sua vez, no estudo conduzido por Rose et al. (1998), a obesidade esteve associada à baixa escolaridade e ao maior número de filhos.

Todavia, no presente estudo, não foi possível verificar associação significativa entre a escolaridade e o estado nutricional dos trabalhadores avaliados. Assim, cabe destacar que, nesta pesquisa, a variável mais associada ao estado nutricional foi à idade, seguida pelo sexo, enquanto a escolaridade não influenciou o estado nutricional dos indivíduos.

No que tange à obesidade abdominal, Castro et al. (2004), ao estudarem o padrão dietético e o estado nutricional de operários de uma empresa metalúrgica, verificaram alta freqüência de sobrepeso $(44,6 \%)$, inesperada para trabalhadores de atividade laboral intensa.

No estudo em questão, $20 \%$ dos metalúrgicos tinham valores de circunferência da cintura maiores que $94 \mathrm{~cm}, 5 \%$ apresentavam circunferência da cintura superior a $102 \mathrm{~cm}$ e todos os trabalhadores com IMC ¡Ý $30 \mathrm{~kg} / \mathrm{m} 2$ tinham circunferência da cintura superior a $94 \mathrm{~cm}$. Apesar de terem sido superiores a estes dados, os achados do presente estudo foram inferiores aos do estudo conduzido por Ewald et al. (2003), que, ao analisarem os fatores de risco para doenças crônicas não transmissíveis entre colaboradores de uma Unidade de Alimentação e Nutrição, constataram obesidade abdominal em 58,06\% dos 
colaboradores, tendo o sobrepeso e a obesidade constituído-se nos estados nutricionais predominantes $(58,07 \%)$.

Em sua pesquisa Souza et al. (2003) encontraram prevalência de obesidade abdominal de 35,1\%.

Ressalva-se que, no presente estudo, apesar de os indivíduos do sexo masculino apresentarem maiores chances de estarem com sobrepeso ou obesos, a prevalência de circunferência da cintura elevada foi significativamente superior no sexo feminino.

Os resultados desta pesquisa no que se refere à prevalência de pressão arterial elevada estão de acordo com a média nacional descrita, que corresponde a $15 \pm 3 \%$ da população de vinte anos ou mais de idade 31, e inferiores aos de outros estudos populacionais realizados no Brasil.

Souza et al. (2003) verificaram prevalência de 29,5\% de hipertensão arterial; no estudo de Gigante et al. (1997), a hipertensão arterial foi referida por $22 \%$ dos entrevistados.

A importância relativa das PAS e PAD já foi objeto de inúmeras pesquisas, sendo recentemente destacada a importância da PAS, aconselhando-se, assim, a sua redução. O VII Joint National Committee enfatiza que o risco de desenvolver doença cardíaca ou acidente vascular cerebral aumenta consideravelmente quando a PAS apresenta-se elevada e a PAD apresenta-se normal. (CHOBANIAN, 2003).

No que diz respeito à prevalência de diabetes mellitus II encontrada no presente estudo, esta foi semelhante à do estudo de Matos et al. (2004), no qual foi observada prevalência de 2,5\%, ambos resultados inferiores ao observado na literatura.

A prevalência de diabetes mellitus encontrada no estudo de Souza et al. (2003) foi de 8\%; no estudo de Gigante et al. (1997), 5\% dos entrevistados referiram diabetes. Schaan et al. (2004) observaram prevalência de 12,4\% de diabetes mellitus e 7,4\% de alteração na glicemia de jejum em estudo conduzido com amostra representativa da população adulta do Rio Grande do Sul. Em Ribeirão Preto, São Paulo, Torquato et al. (2003) encontraram prevalências de $12,1 \%$ de diabetes e de 7,7\% de tolerância à glicose alterada.

A hipertensão arterial e a resistência à insulina são associadas à obesidade com muita freqüência. Tanto a PAS quanto a PAD aumentam com o IMC, e os obesos correm mais risco de desenvolver hipertensão do que os indivíduos magros. 
O mesmo ocorre em relação à resistência à insulina e diabetes mellitus tipo II, em que o risco aumenta continuamente com o IMC e diminui com a perda de peso 6. A associação entre a obesidade e a prevalência de hipertensão arterial foi relatada nos trabalhos conduzidos por Gus et al. (2002), estudando uma amostra representativa da população de Porto Alegre, Rio Grande do Sul, e Carneiro et al. (2003), avaliando a influência da distribuição de gordura corporal sobre a prevalência de hipertensão arterial e outros fatores de risco cardiovasculares em indivíduos obesos, semelhantemente aos resultados do presente estudo.

No entanto, apesar de, nesta pesquisa, ter sido observada associação significativa entre o estado nutricional e a pressão arterial com o ajuste para idade, não se verificou associação entre o diabetes mellitus II e a glicemia de jejum alterada.

Em contraposição aos nossos achados, no estudo de Schaan et al. (2004) foi verificada maior prevalência de obesidade entre os indivíduos com diabetes ou alteração na glicemia de jejum. Ortiz e Zanetti (2000), em estudo realizado com trabalhadores de uma instituição de ensino superior, encontraram a obesidade como freqüente fator de risco para o diabetes mellitus.

Os primeiros estudos sobre a composição corporal e distribuição da gordura corporal para identificar indivíduos com um risco maior para doenças cardiovasculares e diabetes mellitus II utilizaram, principalmente, o IMC e a relação cintura-quadril. (COUTINHO, 2001). Contudo, segundo Zhu et al. (2005), a circunferência da cintura per se é capaz de refletir a magnitude dos depósitos abdominais de tecido adiposo bem como a gordura total, complementando, assim, o índice de massa corporal na avaliação dos riscos de doença cardiovascular associados à obesidade pelo fornecimento de uma medida da distribuição da gordura corporal, além de ser uma medida antropométrica simples e clinicamente útil.

Os resultados deste estudo mostraram associação significativa entre a classificação da circunferência da cintura e as prevalências de PAS e PAD elevadas, sem variação na razão de prevalências das duas variáveis. Houve ainda uma tendência de as razões de prevalência para a circunferência de a cintura serem maiores do que aquelas para o estado nutricional. Semelhantemente, Lerário et al. (2002), avaliando as implicações do excesso de peso em nipo-brasileiros, constataram que, nos grupos com excesso de peso ou adiposidade central, as taxas de hipertensão arterial foram sempre superiores. 
De acordo com Simone et al. (2005), a composição corporal e a distribuição de gordura podem influenciar a hemodinâmica sistêmica em adultos normotensos e hipertensos de uma amostra populacional, mesmo na ausência de obesidade.

Neste estudo verifica-se que o excesso de gordura abdominal não esteve associado à presença de diabetes mellitus II ou alteração na glicemia de jejum. Em um trabalho conduzido por Kim et al. (2004) avaliando a gordura visceral com um índice alternativo para estimar o risco de doenças cardiovasculares e outras doenças metabólicas, quando a amostra foi dividida em tercis de acordo com a espessura da gordura visceral total, não se encontrou diferença significante nas concentrações de glicemia de jejum ou de hemoglobina glicosilada entre os grupos. Souza et al. (2003), portanto observaram, em seu estudo, maior prevalência de diabetes mellitus entre obesos ou com excesso de gordura abdominal na população avaliada.

Em seu estudo Trindade et al. (1998) com população urbana de Passo Fundo, Rio Grande do Sul, e Schaan et al. (2004), analisando os fatores de risco cardiovascular no diabetes mellitus e na glicemia de jejum alterada, observaram associação significante entre a prevalência de hipertensão arterial sistêmica e de diabetes mellitus/alteração na glicemia de jejum, similarmente aos resultados obtidos no presente trabalho.

De acordo com os achados deste estudo permitiram a realização de um diagnóstico precoce do estado nutricional e de fatores de risco para doenças crônicas não transmissíveis na população avaliada.

Possibilitou-se, então, a modificação destes por meio de intervenções de cunho preventivo e educacional promovidas pela empresa, conscientizando os colaboradores sobre a importância de hábitos alimentares saudáveis e promovendo, assim, melhor qualidade de vida e maior produtividade.

\section{REFERÊNCIAS}

BLUMENKRANTZ, M. Obesity: the world's metabolic disorder. Disponível: <http://www. quantumhcp. com.obesity.htm>. Acesso em: 01.mar. 2006.

BRASIL. Ministério da Saúde. Secretaria de Políticas Públicas de Saúde. Programa Nacional de promoção da atividade física "Agita Brasil". Atividade física e sua contribuição para a qualidade de vida Rev. Saúde Pública, v. 36, p. 254-256, 2002.

Conexões: revista da Faculdade de Educação Física da UNICAMP, Campinas, v. 6, n. 1, p. 14-30, jan/abr. 2008 
CAMERON, N. The measurement of human growth. Sydney: Croom Helm; 1984.

CARNEIRO, G. et al. Consenso latino-americano em obesidade, 2001. Disponível: <http://www. abeso. org.br/consenso.doc >. Acesso em 02. out. 2006.

CASTANHEIRA, M.; OLINTO, M. T. A.; GIGANTE, D. P. Associação de variáveis sócio-demográficas e comportamentais com a gordura abdominal em adultos: estudo de base populacional no Sul do Brasil. Cad. Saúde Pública, v. 19 supl. 1, p. 555-65, 2003.

CAStro, M. B. T.; ANJOS, L .A.; LOURENÇO, P. M. Padrão dietético e estado nutricional de operários de uma empresa metalúrgica do Rio de Janeiro: Cad Saúde Pública, v. 20, p. 926-934, 2004. CHOBANIAN, A.V. et al. Seventh report of the Joint National Committee on Prevention, Detection, Evaluation, and Treatment of High Blood Pressure. Hypertension, v. 42, p. 1206-1252, 2003.

CERVATO, A. M.; et al. Dieta habitual e fatores de risco para doenças cardiovasculares. Rev Saúde Pública, 31:227-35, 1997.

CRETANI, M. Prevalência de hipertensão arterial sistêmica na população urbana de Passo Fundo (RS). Arq Bras Cardiol, v. 71, p. 127-130, 1998.

DUNCAN, B. B. et al. Fatores de risco para doenças não-transmissíveis em áreas metropolitanas na região sul do Brasil: prevalência e simultaneidade. Rev Saúde Pública, v. 27, p. 143-148, 1993.

EWALD, D. et al. Fatores de risco para doenças crônicas não-transmissíveis entre colaboradores internos de Unidade de Alimentação e Nutrição. In: SIMPÓSIO INTERNACIONAL SOBRE OBESIDADE, 2003. ANAIS... Florianópolis: Universidade Federal de Santa Catarina, 2003. p. 13.

FERREIRA, S. R. G. Influência da distribuição da gordura corporal sobre a prevalência de hipertensão arterial e outros fatores de risco cardiovascular em individuos obesos. Rev Assoc Med Bras, 49:306-11, 2003.

GIGANTE, D. P. et al. Prevalência de obesidade em adultos e seus fatores de risco. Rev Saúde Pública, v. 31, p. 236-246, 1997.

GUS, I.; FISCHMANN, A.; MEDINA, C. Prevalência dos fatores de risco da doença coronariana no Estado do Rio Grande do Sul. Arq Bras Cardiol, v. 78, p. 478-483, 2002.

HAN, T. S. et. Waist circumference action levels in the identification of cardiovascular disease risk factors: prevalence study in a random sample. BMJ, v. 311, p. 1401-1405, 1995.

INSTITUTO Brasileiro de Geografia e Estatística. Pesquisa de orçamentos familiares: POF 2002-2003. Disponível em: <http://www.ibge.gov.br> Acesso em: 05. jan. 2006.

JUNG, R.T. Obesity as a disease. Br Med Bull, v. 53, p. 307-321, 1997. 
JOINT National Committee. The 1988 report of the Joint National Committee on detection, evaluation, and treatment of high blood pressure. Arch Intern Med, v. 148, p. 1023-1038, 1988.

KIM, S. K. et al. Visceral fat thickness measured by ultrasonography can estimate not only visceral obesity but also risks of cardiovascular and metabolic diseases. Am J Clin Nutr, v. 79, p. 593-599, 2004.

LERARIO, D. D. G. ; et al. Excesso de peso e gordura abdominal para a síndrome metabólica em nipobrasileiros. Rev Saúde Pública, 36:4-11, 2002.

MACHADO, P. A. N.; SICHIERI, R. Relação cintura-quadril e fatores de dieta em adultos. Rev Saúde Pública, v. 36, p. 198-204, 2002.

MATOS, M. F. D. et al. Prevalência dos fatores de risco para doença cardiovascular em funcionários do centro de pesquisas da Petrobrás. Arq Bras Cardiol, v. 82, p. 1- 4, 2004.

MONTEIRO, C. A. et al. Da desnutrição para a obesidade: a transição nutricional no Brasil. In: MONTEIRO, C. A. (Org.). Velhos e novos males da saúde no Brasil: a evolução do país e de suas doenças. São Paulo: Hucitec, 1995. p. 247-255.

ORGANIZAÇÃO Mundial da Saúde. Obesidade: prevenindo e controlando a epidemia global. São Paulo: Roca, 2004.

ORTIZ, M. C. A.; ZANETTI, M. L. Diabetes mellitus: fatores de risco em uma instituição de ensino da área da saúde. Rev Latino-Am Enfermagem, v. 8, p. 28-32, 2000.

ROSE, K. M. et al. Genetic and behavioral determinants of waist-hip and waist circumference in women twins. Obes Res, v. 6, p. 383 - 392, 1998.

SANTANA, V. S. et al. Morbidade em candidatos a emprego na região metropolitana de Salvador, Bahia, Brasil. Cad Saúde Pública, v. 17, p. 107 - 115, 2001.

SARTORELLI, D. S.; FRANCO, L. J. Tendências do diabetes mellitus no Brasil: o papel da transição nutricional. Cad Saúde Pública v. 19, supl. 1, p. 29- 36.

SHAAN, B. D.; HARZHEIM, E.; GUS, I. Perfil de risco cardíaco no diabetes mellitus e na glicemia de jejum alterada. Saúde Pública v. 38, p. 529-536, 2004.

SIMONE, G. et al. Body composition and fat distribution influence systemic hemodynamics in the absence of obesity: the HyperGEN Study. Am J Clin Nutr, v. 81, p. 757 - 761. 2005.

SOCIEDADE Brasileira de Cardiologia. Exercício anti-sedentarismo/obesidade. Disponível em: $<$ http://www.cardiol.cbr/funcor/epide/exerc. htm> Acesso em : 01 mar. 2005.

SOCIEDADE Brasileira de Nefrologia. Consenso Brasileiro de hipertensão arterial. $<$ http://www.sbn.org.br/consiiiO.htm> Acesso em: 01. mai. 2005., 1998. 
SOCIEDADE Brasileira de Hipertensão. IV diretrizes brasileiras de hipertensão arterial, 2002. Disponível em: <http://www.sbh.org.br> Acesso em: 22. abr. 2006.

SOCIEDADE Brasileira de Diabetes. Consenso brasileiro sobre diabetes: diagnóstico e classificação do diabetes melito e tratamento do diabetes melito tipo 2. Rio de Janeiro: Diagraphic, 2002.

SOUZA, L. J. et al. Prevalência de obesidade e fatores de risco cardiovascular em Campos, Rio de Janeiro: Arq Bras Endocrinol Metab, v. 47, p. 669-676, 2003.

TORQUATO, M. T. C. G. et al. Prevalence of diabetes mellitus and impaired glucose tolerance in the urban population aged 30-69 years in Ribeirão Preto, Brazil. Méd. J. v. 121, p. 224-230, 2003.

TRINDADE, I. S. et al. Sex differences in the association of socioeconomic status with obesity. Am J Public Health, v. 92, p. 1299 - 1234, 2002.

WORLD Health Organization. Obesity: preventing and managing the global epidemic of a WHO consultation on obesity. Geneva: World Health Organization; 1998.

ZAMAI, C. A. et al. Estudo do conhecimento x incidência de fatores de risco entre escolares do ensino fundamental e médio. Revista Movimento e Percepção, v. 4, n. 5, 2004.

. Atividade física, saúde e doenças crônicas degenerativas: avaliação do nível de conhecimento entre escolares de Campinas. Revista Movimento e Percepção, v. 5, n. 7, 2005.

- Análise do nível de atividade física de estudantes do curso de Educação Física da Universidade Paulista. Revista Movimento e Percepção, v. 8, n. 11, 217-26, 2007.

ZHU, S. et al. Race-ethnicity-specific waist circumference cutoffs for identifying cardiovascular disease risk factors. Am J Clin Nutr, v. 81, p. 409-415, 2005. 


\section{Carlos A. Zamai}

Universidade Paulista

\section{Alan A. Rodrigues \\ Universidade Paulista}

\section{Antonia D. P. Bankoff \\ UNICAMP}

\section{Marcelo Filocomo \\ Universidade Paulista}

\section{Luiz Ernani S. Braga}

Universidade Paulista

\section{José Antonio S. Barbosa \\ Pontifícia Universidade Católica de Campinas}

\section{Referência do artigo:}

\section{ABNT}

ZAMAI, C. A. et. al. Estudo dos fatores de risco para o desenvolvimento de doenças crônicas não transmissíveis entre funcionários. Conexões, v. 6, n. 1, p. 14-30, 2008.

\section{APA}

Zamai, C. A., Rodrigues, A. A., Bankoff, A. D. P., Filocomo, M., Braga, L. E. S., \& Barbosa, J. A. S. (2008). Estudo dos fatores de risco para o desenvolvimento de doenças crônicas não transmissíveis entre funcionários. Conexões, 6(1), 14-30.

\section{VANCOUVER}

Zamai CA, Rodrigues AA, Bankoff ADP, Filocomo M, Braga LES, Barbosa JAS. Estudo dos fatores de risco para o desenvolvimento de doenças crônicas não transmissíveis entre funcionários. Conexões, 2008, 6(1): 14-30. 